\title{
The fibrotic process: summary
}

\author{
MALCOLM I. V. JAYSON \\ From the Department of Medicine, University of Bristol, and Royal National Hospital for \\ Rheumatic Diseases, Bath
}

In this symposium the emphasis in papers and in discussion was on the mechanisms of collagen formation and turnover and the factors influencing them. We are beginning to understand some of the fundamental features leading to abnormal fibrosis in disease. Rational explanations of the values of some forms of therapy together with potential new methods of treatment are beginning to emerge.

Macrophages exposed to sublethal doses of asbestos or silica will form fibrogenic factors which stimulate fibroblasts to produce more collagen. Whether these factors are the same as the connective tissue activating peptide described by Castor and the fibrogenic factors found in the liver by McGee is not yet elucidated. It is not clear whether fibrogenic factors act by stimulating individual fibroblasts to produce more collagen or by causing them to divide, so increasing their numbers. As yet there are no suggestions for methods to interfere with the stimulus to fibrogenesis.

In clinical silicosis immunological factors may also be relevant-subjects exposed to silica who develop the most florid lung changes also have the highest antinuclear factor titres. It is also known that silica can interfere with the capacity of cells to mount immune responses. Anticollagen antibodies may have some direct effect on fibroblasts, increasing collagen synthesis. Altered immunity seems important in progressive systemic sclerosis and in the recently described industrial model of that disease, vinyl chloride acro-osteolysis. However, the pathogenesis of vascular changes in these diseases is still not clear.

The sequence of events by which collagen is formed within the fibroblasts has largely been elucidated and lead to the experimental trials of the proline analogues which become incorporated into collagen precursors in an endeavour to interfere with collagen synthesis. Although this works experimentally it is doubtful whether it will be of clinical value, since the abnormal proline analogues may be incorporated into many of the body proteins with a wide range of possible side effects.

Of greater potential value are the experimental observations that colchicine will interact with the microtubules and interfere with the secretion of collagen by fibroblasts. There is some evidence that it may be useful in systemic sclerosis and cirrhosis of the liver. However, this drug has been used for $\omega$ many years for gout and it is surprising that there are no reports of interference with wound healing in such patients. Aminophylline may have similar $\omega^{\circ}$ effects on collagen secretion, but there is no evidence that it protects the lungs against fibrosis.

Once secreted from the fibroblasts the extension peptides are cleaved from the procollagen molecules ? which then undergo cross-linkage to form fibres of high tensile strength. There are two types of cross- 을 links, and they both undergo maturation and stabilization by biochemical processes that are at $T$ present not clear but which render them resistant to chemical attack. Only one of these types of cross-links is susceptible to D-penicillamine, which can block its formation and cleave it before stabilip $\overrightarrow{0}$ ation. Dermal collagen contains this cross-link alons. accounting for the limited success of the drug fot the cutaneous manifestations of systemic sclerosis. The failure to control the visceral manifestations of the disease could be due to visceral collagen containing both types of cross-links. Rheumatoid synovium contains collagen that is not directly affected by penicillamine, so the drug must have some alternative mechanism of action. The value of the drug for hepatic cirrhosis is uncertain. Although BAPN seems more effective than D-penicillamine in inhibiting cross-link formation toxic effects are common and are partly due to lathyritic effects in unwanted situations.

Breakdown of collagen is mediated by two distinct series of enzymes - collagenases and proteases. Collagenases act extracellularly on the collagen molecule cleaving it at a specific site into two fragments which are phagocytozed and then degraded by the proteases. However, there is some evidence of collaboration between collagenases and proteases in the initial breakdown of collagen molecules.

The source of collagenases is of obvious interest They may be produced by macrophages stimulated by lymphokines and perhaps by anticollagen antibodies. Their production may be modulated by hormones, as in the involuting uterus and in the tadpole tail. Collagenase does not have a uniform effect on collagen molecules. There are inhibitors 
of collagenase activity such as $\alpha-1$ antitrypsin and other serological factors. The collagen molecule may be surrounded by a coat of proteoglycan protecting it from enzyme attack. Moreover, the different types of collagen show differing susceptibilities to collagenases. Perhaps herein lies some potential therapy if we can distinguish the unwanted collagen and remove it with appropriate enzymes.

Corticosteroids have proved disappointing in limiting unwanted fibrosis despite their ability to decrease the collagen content of tissues as an undesirable side effect of treatment. Although corticosteroid osteoporosis is often quoted as an example of this, in corticosteroid-treated rheumatoid arthritics it is difficult to distinguish the specific effect on osteoporosis of the corticosteroids from those due to the severity of the underlying disease and resultant immobility. It is not clear whether corticosteroids act principally by inhibiting collagen synthesis, increasing degradation, or by some combination of the two.

Understanding the mechanisms of collagen production and degradation and the changes induced by diseases and chemical agents will surely prove relevant to virtually the whole field of medicine. 